C-A/AP/\#187

January 2005

\title{
Matrix Perturbation Approach to the Weak Linear Coupling
}

Y. Luo

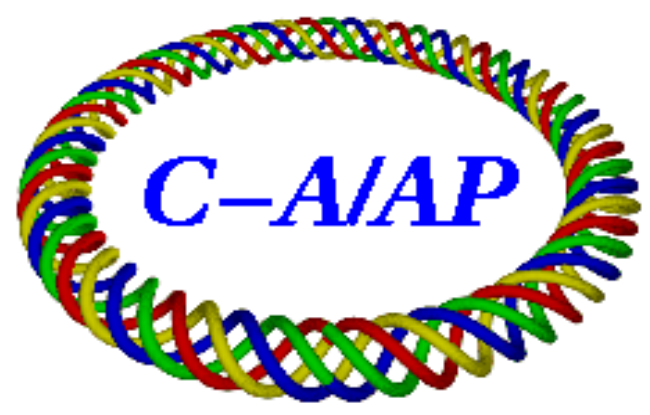

Collider-Accelerator Department Brookhaven National Laboratory Upton, NY 11973 
$\mathrm{CAD} / \mathrm{AP} / 187$

January 20,2005

\title{
Matrix perturbation approach to the weak linear coupling
}

\author{
Y. Luo \\ Brookhaven National Laboratory, Upton, NY 11973, USA
}

Following Peggs and Billing's work [1, 2], the perturbation matrix approach to the weak linear coupling is reset and developed. Two complex quantities, $h_{+}$and $h_{-}$, are defined, that are related to the linear sum and difference coupling resonances, respectively. Based on the perturbation approach, $\overline{\mathbf{H}}, r, \overline{\mathbf{C}}$ in the normalized coordinate system are analytically calculated in different coupling regimes. Six observables of the weak linear difference coupling are defined. Their analytical solutions are obtained. Comparisons ar made between the matrix perturbation approach and the Hamiltonian weak coupling approaches.

\section{Introduction}

Linear betatron coupling was successfully parameterized through the strict matrix approach $[3,4,2,5,6]$, where Twiss and coupling parameters are defined at each point in the ring. The uncoupled motions are obtained through a matrix similarity transformation. The strict matrix approach is valid for strong and weak coupling. However, it is not straightforward to assign analytical expressions to the coupling parameters and the tune shifts for the weak coupling, or for random coupling source distribution situation.

In this article, following Peggs and Billing 's work $[1,2]$, the matrix perturbation approach to weak linear coupling is reset and developed in the normalized coordinate system. First, the one-turn transfer matrix in the normalized coordinated system is derived again and then decoupled. $\overline{\mathbf{H}}, r, \overline{\mathbf{C}}$ in that coordinate system are analytically calculated in different coupling regimes. Six observables of the weak linear difference coupling are defined. Their analytical solutions are obtained. The six observables can be experimentally obtained from the turn-by-turn beam position monitor (BPM) $(x, y)$ data. Comparisons are made between the perturbation matrix approach and the Hamiltonian perturbation approaches.

\section{Strict Matrix approach}

In the section, the strict matrix approach to linear coupling is reviewed. The linear coupling's action-angle parameterization gives the general coordinate expressions. The normalized coordinate system is introduced, and the one-turn transfer matrix in the coordinate system is decoupled, similarly to that in the laboratory system.

\subsection{In the laboratory coordinate system}

The transverse one-turn transfer matrix $\mathbf{T}$ at one point in the ring is

$$
\mathbf{T}=\left(\begin{array}{cc}
\mathbf{M} & \mathbf{m} \\
\mathbf{n} & \mathbf{N}
\end{array}\right),
$$

that can be decoupled based on a matrix similarity transformation $[3,4,2]$,

$$
\mathbf{T}=\mathbf{V}\left(\begin{array}{cc}
\mathbf{A} & \mathbf{0} \\
\mathbf{0} & \mathbf{B}
\end{array}\right) \mathbf{V}^{-\mathbf{1}},
$$

where

$$
\mathbf{V}=\left(\begin{array}{cc}
r \mathbf{I} & \mathbf{C} \\
-\mathbf{C}^{+} & r \mathbf{I}
\end{array}\right)
$$


To easily distinguish Twiss parameter $\gamma$, I use $r$ in $\mathbf{V}$ instead of $\gamma$ used in other literature. $\mathbf{I}$ is $2 \times 2$ unit matrix. $\mathbf{C}$ is the coupling matrix,

$$
\mathbf{C}^{+}=\left(\begin{array}{cc}
c_{22} & -c_{12} \\
-c_{21} & c_{11}
\end{array}\right)
$$

$r$ and $\mathbf{C}$ are connected by $r^{2}+\|\mathbf{C}\|=\mathbf{1}$.

$\mathbf{A}$ and $\mathbf{B}$ define the two uncoupled eigen mode motions. They usually are parameterized to give Twiss parameters according to Courant and Snyder's uncoupled one-dimensional parameterization[7],

$$
\left(\begin{array}{cc}
\cos \left(2 \pi \mu_{i}\right)+\alpha_{i} \sin \left(2 \pi \mu_{i}\right) & \beta_{i} \sin \left(2 \pi \mu_{i}\right) \\
-\gamma_{i} \sin \left(2 \pi \mu_{i}\right) & \cos \left(2 \pi \mu_{i}\right)-\alpha_{i} \sin \left(2 \pi \mu_{i}\right)
\end{array}\right) .
$$

Since the two eigen tunes are

$$
\begin{aligned}
2 \cos \left(2 \pi \mu_{I, I I}\right)= & \frac{1}{2}(\operatorname{Tr} \mathbf{M}+\operatorname{Tr} \mathbf{N}) \\
& \pm \frac{1}{2} \sqrt{(\operatorname{Tr} \mathbf{M}-\operatorname{Tr} \mathbf{N})^{2}+\mathbf{4}\left\|\mathbf{m}^{+}+\mathbf{n}\right\|,}
\end{aligned}
$$

where the sign is chosen assuming that eigen mode I is more related to the horizontal plane than the vertical plane. Therefore,

$$
[\operatorname{Tr}(\mathbf{A}-\mathbf{B})]^{2}=[\operatorname{Tr}(\mathbf{M}-\mathbf{N})]^{2}+4\left\|\mathbf{m}^{+}+\mathbf{n}\right\| .
$$

Defining

$$
\mathbf{H}=\mathbf{m}+\mathbf{n}^{+},
$$

and comparing Eqs. (1) and (2), after some algebra, one obtains $[2,5]$

$$
\begin{gathered}
r=\sqrt{\frac{1}{2}+\frac{1}{2} \sqrt{\frac{[\operatorname{Tr}(\mathbf{M}-\mathbf{N})]^{2}}{[\operatorname{Tr}(\mathbf{M}-\mathbf{N})]^{2}+\mathbf{4}\|\mathbf{H}\|}},} \\
\mathbf{C}=-\frac{\mathbf{H}}{\mathbf{r T r}(\mathbf{A}-\mathbf{B})} .
\end{gathered}
$$

\subsection{The action-angle parameterization [6]}

The linear coupling's action-angle parameterization is convenient for carrying out analytical calculations and for interpetating the experimental turn-by-turn BPM data.

$\mathbf{A}$ and $\mathbf{B}$ in Eq. (2) can be further parameterized like $\mathbf{U}_{\mathbf{i}} \mathbf{R}\left(\mathbf{2} \pi \mu_{\mathbf{i}}\right) \mathbf{U}_{\mathbf{i}}^{-\mathbf{1}}, i=I, I I$, where

$$
\mathbf{U}_{\mathbf{i}}=\left(\begin{array}{cc}
\sqrt{\beta_{i}} & 0 \\
\frac{-\alpha_{i}}{\sqrt{\beta_{i}}} & \frac{1}{\sqrt{\beta_{i}}}
\end{array}\right) .
$$

Then, the one-turn transfer matrix $\mathbf{T}$ is rewritten as

$$
\mathbf{T}=\mathbf{V U R}\left(2 \pi \mu_{\mathbf{I}}, 2 \pi \mu_{\mathbf{I I}}\right) \mathbf{U}^{-1} \mathbf{V}^{-1},
$$

where

$$
\begin{gathered}
\mathbf{U}=\left(\begin{array}{cc}
\mathbf{U}_{\mathbf{I}} & \mathbf{0} \\
\mathbf{0} & \mathbf{U}_{\mathbf{I I}}
\end{array}\right) \\
\mathbf{R}\left(\boldsymbol{\Delta} \boldsymbol{\Phi}_{\mathbf{I}}, \boldsymbol{\Delta} \boldsymbol{\Phi}_{\mathbf{I I}}\right)=\left(\begin{array}{cc}
\mathbf{R}\left(\boldsymbol{\Delta} \boldsymbol{\Phi}_{\mathbf{I}}\right) & \mathbf{0} \\
\mathbf{0} & \mathbf{R}\left(\boldsymbol{\Delta} \boldsymbol{\Phi}_{\mathbf{I I}}\right)
\end{array}\right) \\
\mathbf{R}\left(\boldsymbol{\Delta} \boldsymbol{\Phi}_{\mathbf{i}}\right)=\left(\begin{array}{cc}
\cos \Delta \Phi_{i} & \sin \Delta \Phi_{i} \\
-\sin \Delta \Phi_{i} & \cos \Delta \Phi_{i}
\end{array}\right)
\end{gathered}
$$

According to the action-angle parameterization, the particle's coordinate $\mathbf{X}=\left(\mathbf{x}, \mathbf{x}^{\prime}, \mathbf{y}, \mathbf{y}^{\prime}\right)$ in the laboratory coordinate system is

$$
\mathbf{X}=\mathbf{P}\left(\begin{array}{c}
\sqrt{2 J_{I}} \cos \Phi_{I} \\
-\sqrt{2 J_{I}} \sin \Phi_{I} \\
\sqrt{2 J_{I I}} \cos \Phi_{I I} \\
-\sqrt{2 J_{I I}} \sin \Phi_{I I}
\end{array}\right)
$$


where $J_{I, I I}$ are the globally constant actions of the two eigen modes, and $\Phi_{I, I I}$ are the eigen mode phases.

$\mathbf{P}$ can be obtained analytically or experimentally. If it is described in Twiss and coupling parameters defined above,

$$
\mathbf{P}=\mathbf{V U}
$$

One can prove that $p_{12}=0, p_{34}=0$.

\subsection{In the normalized coordinate system}

Defining the normalized coordinate $\overline{\mathbf{X}}$ as

$$
\overline{\mathbf{X}}=\mathbf{U}^{-1} \mathbf{X}
$$

then according to Eq. (16),

$$
\overline{\mathbf{X}}=\overline{\mathbf{V}}\left(\begin{array}{c}
\sqrt{2 J_{I}} \cos \Phi_{I} \\
-\sqrt{2 J_{I}} \sin \Phi_{I} \\
\sqrt{2 J_{I I}} \cos \Phi_{I I} \\
-\sqrt{2 J_{I I}} \sin \Phi_{I I}
\end{array}\right)
$$

where

$$
\overline{\mathbf{V}}=\mathbf{U}^{-1} \mathbf{V U} .
$$

The transfer matrix $\overline{\mathbf{T}}_{1 \rightarrow 2}$ in the normalized coordinate system is

$$
\overline{\mathbf{T}}_{1 \rightarrow 2}=\mathbf{U}_{\mathbf{2}}^{-\mathbf{1}} \mathbf{T}_{\mathbf{1} \rightarrow \mathbf{2}} \mathbf{U}_{\mathbf{1}},
$$

where $\mathbf{U}_{\mathbf{1}}$ and $\mathbf{U}_{\mathbf{2}}$ are the $\mathbf{U}$ ( Eq. (13) ) at point 1 and point 2, respectively. In uncoupled situation, it is reduced to

$$
\overline{\mathbf{T}}_{1 \rightarrow 2}=\mathbf{R}\left(\boldsymbol{\Delta} \phi_{\mathbf{x}}, \boldsymbol{\Delta} \phi_{\mathbf{y}}\right),
$$

where $\Delta \phi_{x}$ and $\Delta \phi_{y}$ are the horizontal and vertical phase advances between the two points. The one-turn transfer matrix $\overline{\mathbf{T}}$ in the normalized coordinate system is

$$
\overline{\mathbf{T}}=\mathbf{U}^{-1} \mathbf{T} \mathbf{U} .
$$

Substituting Eq. (12) into Eq. (23), $\overline{\mathbf{T}}$ is decoupled as in the laboratory coordinate system,

$$
\overline{\mathbf{T}}=\overline{\mathbf{V}} \mathbf{R}\left(\mathbf{2} \pi \mu_{\mathrm{I}}, \mathbf{2} \pi \mu_{\mathrm{II}}\right) \overline{\mathbf{V}}^{-\mathbf{1}},
$$

$\overline{\mathbf{V}}$ is given by Eq.(20), and is rewritten similarly as that in the laboratory coordinate system,

$$
\overline{\mathbf{V}}=\left(\begin{array}{cc}
\bar{r} \mathbf{I} & \overline{\mathbf{C}} \\
-\overline{\mathbf{C}}^{+} & \bar{r} \mathbf{I}
\end{array}\right),
$$

where $\bar{r}^{2}+|| \overline{\mathbf{C}} \mid=1$.

At any point in the ring, substituting Eqs. (3) and (25) into (20), one gets

$$
\begin{aligned}
& \overline{\mathbf{H}}=\mathbf{U}_{\mathbf{I}}^{-1} \mathbf{H} \mathbf{U}_{\mathbf{I I}}, \\
& \overline{\mathbf{C}}=\mathbf{U}_{\mathbf{I}}^{-1} \mathbf{C U}_{\mathbf{I I}} .
\end{aligned}
$$

Therefore,

$$
\begin{aligned}
\|\overline{\mathbf{H}}\| & =\|\mathbf{H}\|, \\
\|\overline{\mathbf{C}}\| & =\|\mathbf{C}\|, \\
\bar{r} & =r .
\end{aligned}
$$

In the following discussion, I do not distinguish between $r$ and $\bar{r}$.

\section{Perturbation Matrix approach}

In this section, the perturbation matrix approach to the weak linear coupling is reset systematically. And two complex quantities, $h_{+}$and $h_{-}$are defined, that are related to the linear sum and difference coupling resonances, respectively. 


\subsection{Perturbed one-turn matrix}

Here the perturbed one-turn transfer matrix in the normalized coordinate system is derived again. According to Eq. (21), the transfer matrix for a thin skew quadrupole in the normalized coordinate system is

$$
\overline{\mathbf{M}}_{s q, j}=\left(\begin{array}{cc}
\mathbf{I} & q_{j} \mathbf{Q} \\
q_{j} \mathbf{Q} & \mathbf{I}
\end{array}\right),
$$

where $q_{j}=\sqrt{\beta_{x, j} \beta_{y, j}}\left(k_{s} d l\right)_{j}, \beta_{x, j}$ and $\beta_{y, j}$ are the unperturbed betatron amplitude functions, $\left(k_{s} d l\right)_{j}$ is the integrated skew quadrupole strength,

$$
\mathbf{Q}=\left(\begin{array}{cc}
0 & 0 \\
1 & 0
\end{array}\right)
$$

So in the normalized coordinate system, the perturbed one-turn transfer matrix at one point is

$$
\overline{\mathbf{T}}=\mathbf{R}_{\mathrm{N}+\mathbf{1}} \overline{\mathbf{M}}_{\mathrm{sq}, \mathrm{N}} \mathbf{R}_{\mathrm{N}} \ldots \overline{\mathrm{M}}_{\mathrm{sq}, 2} \mathbf{R}_{2} \overline{\mathrm{M}}_{\mathrm{sq}, \mathbf{1}} \mathbf{R}_{1},
$$

where $\mathbf{R}_{\mathbf{j}}=\mathbf{R}\left(\boldsymbol{\Delta} \phi_{\mathbf{x}, \mathbf{j}}, \boldsymbol{\Delta} \phi_{\mathbf{y}, \mathbf{j}}\right), \Delta \phi_{x, j}, \Delta \phi_{y, j}$ are the uncoupled horizontal and vertical phase advances between the adjacent skew quadrapoles.

We define a new matrix $\overline{\mathbf{M}}_{s q, j}^{\prime}$, that meets

$$
\overline{\mathbf{M}}_{s q, j} \mathbf{R}\left(\phi_{\mathbf{x}, \mathbf{j}}, \phi_{\mathbf{y}, \mathbf{j}}\right)=\mathbf{R}\left(\phi_{\mathbf{x}, \mathbf{j}}, \phi_{\mathbf{y}, \mathbf{j}}\right) \overline{\mathbf{M}}_{\mathbf{s q}, \mathbf{j}}^{\prime} .
$$

where $\phi_{x, j}, \phi_{y, j}$ are the uncoupled betatron phase advances from the $j$ th skew quadrupole magnet to the reference point at which the one-turn transfer matrix is calculated. Therefore,

$$
\begin{aligned}
& \overline{\mathbf{M}}_{s q, j}^{\prime}=\mathbf{R}^{-\mathbf{1}}\left(\phi_{\mathbf{x}, \mathbf{j}}, \phi_{\mathbf{y}, \mathbf{j}}\right) \overline{\mathbf{M}}_{\mathbf{s q}, \mathbf{j}} \mathbf{R}\left(\phi_{\mathbf{x}, \mathbf{j}}, \phi_{\mathbf{y}, \mathbf{j}}\right) \\
& =\left(\begin{array}{cc}
\mathbf{I} & \mathbf{R}\left(-\phi_{\mathbf{x}, \mathbf{j}}\right) \underset{\mathbf{j}}{\mathbf{q} \mathbf{R}\left(\phi_{\mathbf{y}, \mathbf{j}}\right)}
\end{array}\right),
\end{aligned}
$$

or in compact,

$$
\begin{gathered}
\overline{\mathbf{M}}_{s q, j}^{\prime}=\left(\begin{array}{cc}
\mathbf{I} & \mathbf{F}_{\mathbf{j}} \\
-\mathbf{F}_{\mathbf{j}}^{+} & \mathbf{I}
\end{array}\right), \\
\mathbf{F}_{\mathbf{j}}=\mathbf{R}\left(-\phi_{\mathbf{x}, \mathbf{j}}\right) \mathbf{q}_{\mathbf{j}} \mathbf{Q R}\left(\phi_{\mathbf{y}, \mathbf{j}}\right) .
\end{gathered}
$$

Considering Eq. (34), the one-turn transfer matrix $\overline{\mathbf{T}}$ Eq. (33) is re-written as

$$
\overline{\mathbf{T}}=\mathbf{R}\left(2 \pi \mu_{\mathrm{x}}, \mathbf{2} \pi \mu_{\mathrm{x}}\right) \overline{\mathbf{M}}_{\mathrm{sq}, \mathbf{N}}^{\prime} \overline{\mathbf{M}}_{\mathrm{sq}, \mathbf{N}-\mathbf{1}}^{\prime} \ldots \overline{\mathbf{M}}_{\mathrm{sq}, 2}^{\prime} \overline{\mathbf{M}}_{\mathrm{sq}, \mathbf{1}}^{\prime},
$$

where $\mu_{x, y}$ are the uncoupled horizontal and vertical tunes without any couplers. If we only keep the terms up to the first order of skew quadrupole strength $q_{j}$ s, the one-turn transfer matrix Eq. (38) is approximated as

$$
\begin{aligned}
\overline{\mathbf{T}} & =\left(\begin{array}{cc}
\mathbf{R}_{\mathbf{x}} & \mathbf{0} \\
\mathbf{0} & \mathbf{R}_{\mathbf{y}}
\end{array}\right)\left(\begin{array}{cc}
\mathbf{I} & \mathbf{F} \\
-\mathbf{F}^{+} & \mathbf{I}
\end{array}\right) \\
& =\left(\begin{array}{cc}
\mathbf{R}_{\mathbf{x}} & \mathbf{R}_{\mathbf{x}} \mathbf{F} \\
-\mathbf{R}_{\mathbf{y}} \mathbf{F}^{+} & \mathbf{R}_{\mathbf{y}}
\end{array}\right),
\end{aligned}
$$

where

$$
\mathbf{F}=\sum_{\mathbf{j}=\mathbf{1}}^{\mathbf{N}} \mathbf{F}_{\mathbf{j}} .
$$

For simplicity, hereafter I use $\mathbf{R}_{\mathbf{x}}, \mathbf{R}_{\mathbf{y}}$ to represent $\mathbf{R}\left(\mathbf{2} \pi \mu_{\mathbf{x}}\right), \mathbf{R}\left(\mathbf{2} \pi \mu_{\mathbf{y}}\right)$, respectively.

Two assumptions were used above. One is the thin lens skew quadrupole, the other is the weak coupling that means $q_{j}$ is small for each individual skew quadrupole. Peggs first obtained Eq. (39) in [1] with the projection approach. 


\subsection{Matrix $\overline{\mathbf{H}}$}

Decouple the perturbed one-turn transfer matrix Eq. (39),

$$
\begin{gathered}
r=\sqrt{\frac{1}{2}+\frac{1}{2} \sqrt{\frac{T r\left(\mathbf{R}_{\mathbf{x}}-\mathbf{R}_{\mathbf{y}}\right)^{\mathbf{2}}}{\operatorname{Tr}\left(\mathbf{R}_{\mathbf{x}}-\mathbf{R}_{\mathbf{y}}\right)^{2}+4|| \overline{\mathbf{H}} \|}}}, \\
\overline{\mathbf{C}}=-\frac{\overline{\mathbf{H}}}{r \operatorname{Tr}(\overline{\mathbf{A}}-\overline{\mathbf{B}})},
\end{gathered}
$$

where

$$
\begin{gathered}
\overline{\mathbf{H}}=\mathbf{R}_{\mathbf{x}} \mathbf{F}+\left(-\mathbf{R}_{\mathbf{y}} \mathbf{F}\right)^{+}=\mathbf{R}_{\mathbf{x}} \mathbf{F}-\mathbf{F R}_{\mathbf{y}}^{-\mathbf{1}}, \\
{[\operatorname{Tr}(\overline{\mathbf{A}}-\overline{\mathbf{B}})]^{2}=\left[\operatorname{Tr}\left(\mathbf{R}_{\mathbf{x}}-\mathbf{R}_{\mathbf{y}}\right)\right]^{\mathbf{2}}+4\|\overline{\mathbf{H}}\| .}
\end{gathered}
$$

$\overline{\mathbf{H}}$ can be analytically calculated. Following Billing's suggestion[2], $\overline{\mathbf{H}}$ is rewritten as

$$
\overline{\mathbf{H}}=\sin \pi\left(\mu_{x}+\mu_{y}\right) \overline{\mathbf{H}}_{-}+\sin \pi\left(\mu_{x}-\mu_{y}\right) \overline{\mathbf{H}}_{+} \mathbf{J},
$$

where

$$
\begin{gathered}
\overline{\mathbf{H}}_{-}=\sum_{j=1}^{N} q_{j} \mathbf{R}\left[\pi\left(\mu_{\mathbf{x}}-\mu_{\mathbf{y}}\right)-\left(\phi_{\mathbf{x}, \mathbf{j}}-\phi_{\mathbf{y}, \mathbf{j}}\right)\right], \\
\overline{\mathbf{H}}_{+}=\sum_{j=1}^{N} q_{j} \mathbf{R}\left[\pi\left(\mu_{\mathbf{x}}+\mu_{\mathbf{y}}\right)-\left(\phi_{\mathbf{x}, \mathbf{j}}+\phi_{\mathbf{y}, \mathbf{j}}\right)\right], \\
\mathbf{J}=\left(\begin{array}{cc}
1 & 0 \\
0 & -1
\end{array}\right) .
\end{gathered}
$$

It can be proved that

$$
\|\overline{\mathbf{H}}\|=\sin ^{2} \pi\left(\mu_{x}+\mu_{y}\right)\left\|\overline{\mathbf{H}}_{-}\right\|-\sin ^{2} \pi\left(\mu_{x}-\mu_{y}\right)\left\|\overline{\mathbf{H}}_{+}\right\| .
$$

Here, two complex quantities are defined,

$$
\begin{aligned}
& h_{-}=\sum_{j=1}^{N} q_{j} e^{i\left[\pi\left(\mu_{x}-\mu_{y}\right)-\left(\phi_{x, j}-\phi_{y, j}\right)\right]}, \\
& h_{+}=\sum_{j=1}^{N} q_{j} e^{i\left[\pi\left(\mu_{x}+\mu_{y}\right)-\left(\phi_{x, j}+\phi_{y, j}\right)\right]} .
\end{aligned}
$$

Therefore,

$$
\begin{gathered}
\overline{\mathbf{H}}_{-}=\left(\begin{array}{cc}
\operatorname{Re}\left\{h_{-}\right\} & \operatorname{Im}\left\{h_{-}\right\} \\
-\operatorname{Im}\left\{h_{-}\right\} & \operatorname{Re}\left\{h_{-}\right\}
\end{array}\right), \\
\overline{\mathbf{H}}_{+}=\left(\begin{array}{cc}
\operatorname{Re}\left\{h_{+}\right\} & \operatorname{Im}\left\{h_{+}\right\} \\
-\operatorname{Im}\left\{h_{+}\right\} & \operatorname{Re}\left\{h_{+}\right\}
\end{array}\right), \\
\|\overline{\mathbf{H}}\|=\sin ^{2} \pi\left(\mu_{x}+\mu_{y}\right)\left|h_{-}\right|^{2}-\sin ^{2} \pi\left(\mu_{x}-\mu_{y}\right)\left|h_{+}\right|^{2},
\end{gathered}
$$

where $R e$ and $I m$ take the real and the imaginary parts of a complex number, respectively.

According to Eqs. (50) and (51), if $h_{ \pm}$are calculated at two points in the ring, and there is no coupler between them, then

$$
\begin{aligned}
& h_{-, 2}=h_{-, 1} e^{i\left(\Delta \phi_{x}-\Delta \phi_{y}\right)}, \\
& h_{+, 2}=h_{+, 1} e^{i\left(\Delta \phi_{x}+\Delta \phi_{y}\right)},
\end{aligned}
$$

where $\Delta \phi_{x}, \Delta \phi_{y}$ are the unperturbed horizontal and vertical phase advances. The beam circulates from point 1 to point 2 . If $h_{ \pm}$are calculated just before and after a thin skew quadrupole, one obtains

$$
\begin{aligned}
& \Delta h_{-}=-2 q_{j} \sin \pi\left(\mu_{x}-\mu_{y}\right), \\
& \Delta h_{+}=-2 q_{j} \sin \pi\left(\mu_{x}+\mu_{y}\right) .
\end{aligned}
$$




\section{$3.3 \quad \mathrm{r}$ and $\overline{\mathrm{C}}$}

Knowing $\overline{\mathbf{H}}$, then according to Eqs. (41), (42), $r$ and $\overline{\mathbf{C}}$ can be determined. For weak coupling, from Eq. (44), the determinant of $\overline{\mathbf{H}}$ should be a global constant. Therefore, according to Eq. (41), $r$ is a global constant, too.

Considering Eqs. (50) and (51), according to Eq. (42), we obtain

$\overline{\mathbf{C}}=-\frac{1}{r \operatorname{Tr}(\overline{\mathbf{A}}-\overline{\mathbf{B}})}\left(\begin{array}{cc}\sin \pi\left(\mu_{x}+\mu_{y}\right) \operatorname{Re}\left\{h_{-}\right\}+\sin \pi\left(\mu_{x}-\mu_{y}\right) \operatorname{Re}\left\{h_{+}\right\} & \sin \pi\left(\mu_{x}+\mu_{y}\right) \operatorname{Im}\left\{h_{-}\right\}-\sin \pi\left(\mu_{x}-\mu_{y}\right) \operatorname{Im}\left\{h_{+}\right\} \\ -\sin \pi\left(\mu_{x}+\mu_{y}\right) \operatorname{Im}\left\{h_{-}\right\}-\sin \pi\left(\mu_{x}-\mu_{y}\right) \operatorname{Im}\left\{h_{+}\right\} & \sin \pi\left(\mu_{x}+\mu_{y}\right) \operatorname{Re}\left\{h_{-}\right\}-\sin \pi\left(\mu_{x}-\mu_{y}\right) \operatorname{Re}\left\{h_{+}\right\}\end{array}\right)$.

And from Eq. (59),

$$
\begin{aligned}
& \bar{c}_{11}-\bar{c}_{22}-i\left(\bar{c}_{12}+\bar{c}_{21}\right)=-\frac{2 \sin \pi\left(\mu_{x}-\mu_{y}\right) h_{+}}{r \operatorname{Tr}(\overline{\mathbf{A}}-\overline{\mathbf{B}})}, \\
& \bar{c}_{22}+\bar{c}_{11}+i\left(\bar{c}_{12}-\bar{c}_{21}\right)=-\frac{2 \sin \pi\left(\mu_{x}+\mu_{y}\right) h_{-}}{r \operatorname{Tr}(\overline{\mathbf{A}}-\overline{\mathbf{B}})} .
\end{aligned}
$$

\section{Different weak linear coupling regimes}

\subsection{Stability criterion}

Assuming the thin skew quadrupole and weak coupling, from Eq. (44), one gets

$$
\left(\cos 2 \pi \mu_{I}-\cos 2 \pi \mu_{I I}\right)^{2}=\left(\cos 2 \pi \mu_{x}-\cos 2 \pi \mu_{y}\right)^{2}+\|\overline{\mathbf{H}}\| .
$$

Therefore, the stable condition for the particle motion is

$$
\left(\cos 2 \pi \mu_{x}-\cos 2 \pi \mu_{y}\right)^{2}+\|\overline{\mathbf{H}}\|>0,
$$

that is,

$$
\left(\cos 2 \pi \mu_{x}-\cos 2 \pi \mu_{y}\right)^{2}+\sin ^{2} \pi\left(\mu_{x}+\mu_{y}\right)\left|h_{-}\right|^{2}-\sin ^{2} \pi\left(\mu_{x}-\mu_{y}\right)\left|h_{+}\right|^{2}>0 .
$$

It is clear that $h_{-}$is related to the linear difference coupling, while $h_{+}$is related to the linear sum coupling. When the accelerator is working close to the linear difference coupling resonance $\mu_{x}-\mu_{y}-p=0$, where $p$ is the integer tune split, $h_{-}$is dominant term since the contribution from the individual skew quadrupole to $h_{-}$sums up. While close to the linear sum coupling, $h_{+}$is dominant term.

Therefore, the particle motion is stable when the accelerator is working close to or on the linear difference coupling resonance. However, there is a stop-band for the linear sum difference coupling resonance, which is given next.

\subsection{Linear difference coupling resonance}

When the accelerator is working close enough to the linear difference coupling resonance, we can ignore $h_{+}$, then according to Eqs. (44) and (41),

$$
\begin{aligned}
&|\operatorname{Tr}(\overline{\mathbf{A}}-\overline{\mathbf{B}})|=\sqrt{4\left(\cos 2 \pi \mu_{x}-\cos 2 \pi \mu_{y}\right)^{2}+4 \sin ^{2} \pi\left(\mu_{x}+\mu_{y}\right)\left|h_{-}\right|^{2}}, \\
& r=\sqrt{\frac{1}{2}+\frac{1}{2} \sqrt{\frac{\sin ^{2} \pi\left(\mu_{x}-\mu_{y}\right)}{\sin ^{2} \pi\left(\mu_{x}-\mu_{y}\right)+\frac{1}{4}\left|h_{-}\right|^{2}}}} \\
& \frac{\sqrt{2}}{2} \leq r<1 .
\end{aligned}
$$

And, following Eq. (59), the normalized $\overline{\mathbf{C}}$ is

$$
\overline{\mathbf{C}}=-\frac{\sin \pi\left(\mu_{x}+\mu_{y}\right)}{r \operatorname{Tr}(\overline{\mathbf{A}}-\overline{\mathbf{B}})}\left(\begin{array}{cc}
\operatorname{Re}\left\{h_{-}\right\} & \operatorname{Im}\left\{h_{-}\right\} \\
-\operatorname{Im}\left\{h_{-}\right\} & \operatorname{Re}\left\{h_{-}\right\}
\end{array}\right) .
$$

According to Eqs. (62) and (54), the fractional eigen tune split is

$$
\left(\mu_{I}-\mu_{I I}-p\right)^{2}=\left(\mu_{x}-\mu_{y}-p\right)^{2}+\left(\frac{1}{2 \pi}\left|h_{-}\right|\right)^{2},
$$


the minimum tune split is $\frac{1}{2 \pi}\left|h_{-}\right|$.

If the accelerator is working exactly on the linear difference coupling resonance, where $\mu_{x}-\mu_{y}-p=0$,

$$
\begin{gathered}
\left|\mu_{I}-\mu_{I I}-p\right|=\frac{1}{2 \pi}\left|h_{-}\right|, \\
r=\frac{\sqrt{2}}{2}, \\
\overline{\mathbf{C}}=-\frac{1}{\sqrt{2}\left|h_{-}\right|}\left(\begin{array}{cc}
\operatorname{Re}\left\{h_{-}\right\} & \operatorname{Im}\left\{h_{-}\right\} \\
-\operatorname{Im}\left\{h_{-}\right\} & \operatorname{Re}\left\{h_{-}\right\}
\end{array}\right) .
\end{gathered}
$$

\subsection{Linear sum coupling resonance}

Similarly, when a particle's motion is close to the linear sum coupling resonance, we can ignore $h_{-}$,

$$
\begin{gathered}
|\operatorname{Tr}(\overline{\mathbf{A}}-\overline{\mathbf{B}})|=\sqrt{4\left(\cos 2 \pi \mu_{x}-\cos 2 \pi \mu_{y}\right)^{2}-4 \sin ^{2} \pi\left(\mu_{x}-\mu_{y}\right)\left|h_{+}\right|^{2}}, \\
r=\sqrt{\frac{1}{2}+\frac{1}{2} \sqrt{\frac{\sin ^{2} \pi\left(\mu_{x}+\mu_{y}\right)}{\sin ^{2} \pi\left(\mu_{x}+\mu_{y}\right)-\frac{1}{4}\left|h_{+}\right|^{2}}}} \\
r>1 .
\end{gathered}
$$

And the normalized $\overline{\mathbf{C}}$ is

$$
\overline{\mathbf{C}}=-\frac{\sin \pi\left(\mu_{x}-\mu_{y}\right)}{r \operatorname{Tr}(\overline{\mathbf{A}}-\overline{\mathbf{B}})}\left(\begin{array}{cc}
\operatorname{Re}\left\{h_{+}\right\} & -\operatorname{Im}\left\{h_{+}\right\} \\
-\operatorname{Im}\left\{h_{+}\right\} & -\operatorname{Re}\left\{h_{+}\right\}
\end{array}\right) .
$$

According to Eqs. (62) and (54), we derive

$$
\left(\mu_{I}+\mu_{I I}-p\right)^{2}=\left(\mu_{x}+\mu_{y}-p\right)^{2}-\left(\frac{1}{2 \pi}\left|h_{+}\right|\right)^{2},
$$

where $\mathrm{p}$ is an integer making $\left(\mu_{x}+\mu_{y}-p\right)$ close to zero. The stable condition for the linear sum coupling resonance is

$$
\left|\mu_{x}+\mu_{y}-p\right|>\frac{1}{2 \pi}\left|h_{+}\right|,
$$

Therefore, $\frac{1}{2 \pi}\left|h_{+}\right|$is the stop-band for the linear sum coupling resonance.

\subsection{Far away from the linear coupling resonances}

When the accelerator is working at distance away from the linear coupling resonances, $h_{-}$and $h_{+}$are both small, and so is $\|\overline{\mathbf{H}}\|$. Therefore,

$$
\begin{aligned}
\operatorname{Tr}(\overline{\mathbf{A}}-\overline{\mathbf{B}})=\operatorname{Tr}\left(\mathbf{R}_{\mathbf{x}}-\mathbf{R}_{\mathbf{y}}\right) & =\mathbf{4} \sin \pi\left(\mu_{\mathbf{x}}+\mu_{\mathbf{y}}\right) \sin \pi\left(\mu_{\mathbf{x}}-\mu_{\mathbf{y}}\right), \\
r & =1 .
\end{aligned}
$$

The normalized $\overline{\mathbf{C}}$ is

$$
\overline{\mathbf{C}}=-\frac{1}{4}\left(\begin{array}{cc}
\frac{\operatorname{Re}\left\{h_{-}\right\}}{\sin \pi\left(\mu_{x}-\mu_{y}\right)}+\frac{\operatorname{Re}\left\{h_{+}\right\}}{\sin \pi\left(\mu_{x}+\mu_{y}\right)} & \frac{\operatorname{Im}\left\{h_{-}\right\}}{\sin \pi\left(\mu_{x}-\mu_{y}\right)}-\frac{\operatorname{Im}\left\{h_{+}\right\}}{\sin \pi\left(\mu_{x}+\mu_{y}\right)} \\
-\frac{\operatorname{Im}\left\{h_{-}\right\}}{\sin \pi\left(\mu_{x}-\mu_{y}\right)}-\frac{\operatorname{Im}\left\{h_{+}\right\}}{\sin \pi\left(\mu_{x}+\mu_{y}\right)} & \frac{\operatorname{Re}\left\{h_{-}\right\}}{\sin \pi\left(\mu_{x}-\mu_{y}\right)}-\frac{\operatorname{Re}\left\{h_{+}\right\}}{\sin \pi\left(\mu_{x}+\mu_{y}\right)}
\end{array}\right) .
$$

\section{$5 \quad$ Difference coupling observables}

Beside the two eigen tunes, another four quantities are defined as the weak different coupling observables. Their analytical solutions are given. They play very important roles in the linear difference coupling observations and imply possible global decoupling loops for the weak linear difference coupling. 


\subsection{6 coupling observables}

The general BPM $(x, y)$ data are written as

$$
\left\{\begin{array}{ccc}
x_{n}= & A_{I, x} \cos \left[2 \pi \mu_{I}(n-1)+\phi_{I, x, 0}\right] \\
& +A_{I I, x} \cos \left[2 \pi \mu_{I I}(n-1)+\phi_{I I, x, 0}\right] \\
y_{n}= & A_{I, y} \cos \left[2 \pi \mu_{I}(n-1)+\phi_{I, y, 0}\right] \\
& +A_{I I, y} \cos \left[2 \pi \mu_{I I}(n-1)+\phi_{I I, y, 0}\right]
\end{array} .\right.
$$

$A_{i, x}$ or $A_{i, y}$ are mode $i$ 's contribution to the $x$ or $y$ plane, respectively. They are non-negative numbers. $\phi_{i, x}$ or $y, 0$ are the initial phases.

The tune split Eq. (69) usually is used for the global linear difference coupling measurement and correction purposes. Besides the two eigen tunes $\mu_{I}$ and $\mu_{I I}$, here another four observables are defined: two amplitude ratios and two phase differences,

$$
\begin{gathered}
\left\{\begin{array}{c}
R_{I}=\frac{A_{I, y}}{A_{I, x}} \\
R_{I I}=\frac{A_{I I, x}}{A_{I I, y}}
\end{array}\right. \\
\left\{\begin{array}{c}
\Delta \phi_{I, 0}=\phi_{I, y, 0}-\phi_{I, x, 0} \\
\Delta \phi_{I I, 0}=\phi_{I I, x, 0}-\phi_{I I, y, 0}
\end{array} .\right.
\end{gathered}
$$

In the uncoupled situation, the values of $R_{I}, R_{I I}$ equal zero, and $\Delta \phi_{I, 0}$ and $\Delta \phi_{I I, 0}$ have no meaning.

\subsection{Analytical solution}

According to Eqs. (18) and (16), together with the uncoupled Twiss parameters, the $(x, y)$ coordinates are

$$
\left\{\begin{array}{rl}
x= & r \sqrt{\beta_{x}} \sqrt{2 J_{I}} \cos \Phi_{I} \\
& +\bar{c}_{11} \sqrt{\beta_{x}} \sqrt{2 J_{I I}} \cos \Phi_{I I}-\bar{c}_{12} \sqrt{\beta_{x}} \sqrt{2 J_{I I}} \sin \Phi_{I I} \\
y= & -\bar{c}_{22} \sqrt{\beta_{y}} \sqrt{2 J_{I}} \cos \Phi_{I}-\bar{c}_{12} \sqrt{\beta_{y}} \sqrt{2 J_{I}} \sin \Phi_{I} \\
& +r \sqrt{\beta_{y}} \sqrt{2 J_{I I}} \cos \Phi_{I I}
\end{array} .\right.
$$

Therefore, the amplitude ratios and the phase differences are

$$
\begin{gathered}
\left\{\begin{array}{c}
R_{I}=\sqrt{\frac{\beta_{y}}{\beta_{x}}} \frac{\sqrt{\bar{c}_{22}^{2}+\bar{c}_{12}^{2}}}{r} \\
R_{I I}=\sqrt{\frac{\beta_{x}}{\beta_{y}}} \frac{\sqrt{\bar{c}_{11}^{2}+\bar{c}_{12}^{2}}}{r}
\end{array}\right. \\
\left\{\begin{array}{c}
\Delta \phi_{I, 0}=-\arctan \frac{\bar{c}_{12}}{\bar{c}_{22}} \\
\Delta \phi_{I I, 0}=\arctan \frac{\bar{c}_{12}}{\bar{c}_{11}}
\end{array}\right.
\end{gathered}
$$

For the linear weak difference coupling, considering Eq. (68), one gets

$$
\begin{gathered}
\left\{\begin{array}{c}
R_{I}=\sqrt{\frac{\beta_{y}}{\beta_{x}}} \frac{\sqrt{1-r^{2}}}{r} \\
R_{I I}=\sqrt{\frac{\beta_{x}}{\beta_{y}}} \frac{\sqrt{1-r^{2}}}{r}
\end{array}\right. \\
\left\{\begin{array}{ccc}
\Delta \phi_{I, 0} & = & \pi-\chi \\
\Delta \phi_{I I, 0} & = & \chi
\end{array}\right.
\end{gathered}
$$

where $\chi$ is $h_{-}$'s phase, $h_{-}=\left|h_{-}\right| e^{i \chi}$. Defining

$$
R=R_{I} R_{I I},
$$

then

$$
R=\frac{1-r^{2}}{r^{2}}
$$


The tune split Eq. (69), the amplitude ratios Eq. (91) and the phase differences Eq. (89) play important roles in the linear difference coupling observations. They imply three possible loops for the global decoupling [8]. In [8], the analytical solutions to the six weak linear difference coupling observables are obtained through the Hamiltonian perturbation theory[9, 10, 11]. C. Gardner also obtained similar expressions for the amplitude ratios and the phase differences through the direct matrix approximation under the weak coupling [12].

\section{Comparison to weak coupling's Hamiltonian approaches}

Guignard developed the Hamiltonian perturbation theory for the linear coupling $[9,10,11]$. By isolating the leading linear sum or difference coupling resonance, the eigen tunes are calculated through the defined coupling coefficients, which are defined as

$$
C^{ \pm}=\frac{1}{2 \pi} \int_{0}^{L} \sqrt{\beta_{x} \beta_{y}} k_{s} e^{i\left[\phi_{x} \pm \phi_{y}-2 \pi \Delta \cdot s / L\right]} d l,
$$

where $\Delta=\mu_{x} \pm \mu_{y}-p, p$ s are integers which make $\Delta$ s close to zero. Comparing this to the definitions of $h_{ \pm}$, it is found that $C^{ \pm}$only includes the leading sum or difference coupling resonance. When the accelerator is exactly working on the linear coupling resonances,

$$
C_{ \pm}=\frac{1}{2 \pi} h_{ \pm}^{*}
$$

$h_{ \pm}^{*}$ are $h_{ \pm}$'s conjugates.

The weak linear coupling's Hamiltonian perturbation theory is widely used for the global coupling measurement and correction. However, this approach treats the sum and difference coupling resonance separately. It works close to the weak coupling resonances, and is not convenient for the turn-by-turn BPM data interpretation.

Bartolini and Schmidt proposed another Hamiltonian approach to the weak linear coupling in [13]. Through the perturbed Hamiltonian Normal-Form, the turn-by-turn normalized coordinates are expressed in the driving terms. These driving terms for linear coupling are

$$
f_{1001,1010}=-\frac{1}{4\left[1-e^{i 2 \pi\left(\mu_{x} \mp \mu_{y}\right)}\right]} \sum_{j=1}^{N}\left(k_{s} d l\right)_{j} \sqrt{\beta_{x, j} \beta_{y, j}} e^{i\left(\phi_{x, j} \mp \phi_{y, j}\right)},
$$

where $f_{1001,1010}$ are related to the linear difference and sum couplings, respectively. This approach is used experimentally at the sextupole and skew quadrupole locations with the turn-by-turn BPM data [14]. The perturbed Hamiltonian Normal-Form works away from the resonances [13].

\section{Acknowledgments}

The author thanks S. Peggs for discussions about the global coupling observables, and thank C. Gardner for those on the matrix approximate approaches. The author also would like thank D. Trobjevic, T. Roser for their help. This work is supported by U.S. DOE under contract No. DE-AC02-98CH10886.

\section{References}

[1] S. Peggs, IEEE Trans. Nucl. Sci. 30, 2460 (1983).

[2] M. Billing, Cornell Report CBN 85-2 ( 1985 ).

[3] L.C. Teng, NAL Report FN-229, 1971.

[4] D. Edwards and L. Teng, IEEE Trans. Nucl. Sci. 20, 3 (1973).

[5] D. Sagan and D. Rubin, Phys. Rev. ST Accel. Beam 2, 074001 (1999).

[6] Y. Luo, Phys. Rev. ST Accel. Beam 7, 124001 (2004). 
[7] E.D. Courant and H.S. Snyder, Ann. Phys. 3 (1958).

[8] Y. Luo, P. Cameron, S. Peggs and D. Trobjevic, Possile phase loop for the global decoupling, BNL C-AD/AP Note 174, Sept. 2004, (unpublished).

[9] G. Guignard, CERN Report No. 76-06, 1976.

[10] G. Guignard, Phys. Rev. E 51, p6104, 1995.

[11] H. Wiedemann, Particle Accelerator Physics II, Nonlinear and Higher-Order Beam Dynamics, SpringerVerlag, 1995.

[12] C. Gardner, Some useful linear coupling approximation, BNL C-AD/AP Note 101, July, 2003, (unpublished).

[13] R. Bartolini and F. Schmidt, Part. Accel. 59, p93-106, 1998.

[14] R. Tomas, PH.D thesis, Direct measurement of resonace driving terms in the Super Proton Sychrotron (SPS) of CERN using beam position monitors, Universitat de Valencia, Spain, 2002. 